\title{
Genetic contribution to postpartum haemorrhage in Swedish population: cohort study of 466686 births
}

\author{
(1) $(\Theta$ OPEN ACCESS
}

\author{
Anna Sara Oberg research fellow ${ }^{12}$, Sonia Hernandéz-Diaź associate professor ${ }^{1}$, Thomas Frisell \\ researcher ${ }^{3}$, Michael F Greene professor ${ }^{4}$, Catarina Almqvist professor ${ }^{25}$, Brian T Bateman assistant \\ professor $^{67}$
}

\begin{abstract}
${ }^{1}$ Department of Epidemiology, Harvard School of Public Health, Boston, MA 02115, USA ; ${ }^{2}$ Department of Medical Epidemiology and Biostatistics, Karolinska Institutet, PO-box 281, S-171 77 Stockholm, Sweden ; ${ }^{3}$ Clinical Epidemiology Unit, Department of Medicine, Karolinska University Hospital, Eugeniahemmet T2, S-171 76 Stockholm, Sweden; ${ }^{4}$ Department of Obstetrics and Gynecology, Massachusetts General Hospital, Harvard Medical School, Boston, MA 02114, USA ; ${ }^{5}$ Astrid Lindgren Children's Hospital, Lung and Allergy Unit, Karolinska University Hospital, S-171 76 Stockholm, Sweden; ${ }^{6}$ Division of Pharmacoepidemiology and Pharmacoeconomics, Department of Medicine, Brigham and Women's Hospital and Harvard Medical School, Boston, MA 02115, USA; ${ }^{7}$ Department of Anesthesia, Critical Care, and Pain Medicine, Massachusetts General Hospital and Harvard Medical School, Boston, MA 02114, USA
\end{abstract}

\begin{abstract}
Objective To investigate the familial clustering of postpartum haemorrhage in the Swedish population, and to quantify the relative contributions of genetic and environmental effects.

Design Register based cohort study.

Setting Swedish population (multi-generation and medical birth registers).

Main outcome measure Postpartum haemorrhage, defined as $>1000$ $\mathrm{mL}$ estimated blood loss.

Participants The first two live births to individuals in Sweden in 1997-2009 contributed to clusters representing intact couples $(n=366$ 350 births), mothers with separate partners ( $n=53292)$, fathers with separate partners $(n=47054)$, sister pairs $(n=97228)$, brother pairs ( $n=91168)$, and mixed sibling pairs $(n=177944)$.
\end{abstract}

Methods Familial clustering was quantified through cluster specific tetrachoric correlation coefficients, and the influence of potential sharing of known risk factors was evaluated with alternating logistic regression. Relative contributions of genetic and environmental effects to the variation in liability for postpartum haemorrhage were quantified with generalised linear mixed models.

Results The overall prevalence of postpartum haemorrhage after vaginal deliveries in our sample was 4.6\%. Among vaginal deliveries, 18\% (95\% confidence interval $9 \%$ to $26 \%$ ) of the variation in postpartum haemorrhage liability was attributed to maternal genetic factors, $10 \%$ ( $1 \%$ to $19 \%$ ) to unique maternal environment, and $11 \%(0 \%$ to $26 \%$ ) to fetal genetic effects. Adjustment for known risk factors only partially explained estimates of familial clustering, suggesting that the observed shared genetic and environmental effects operate in part through pathways independent of known risk factors. There were similar patterns of familial clustering for both of the main subtypes examined (atony and retained placenta), though strongest for haemorrhage after retained placenta.

Conclusions There is a maternal genetic predisposition to postpartum haemorrhage, but more than half of the total variation in liability is attributable to factors that are not shared in families.

\section{Introduction}

Postpartum haemorrhage is a leading cause of maternal mortality in both the developing and developed world. ${ }^{12}$ Most often postpartum haemorrhage is associated with uterine atony, retained placenta, or lacerations/trauma. ${ }^{3}$ While several important risk factors have been identified, including a history of postpartum haemorrhage or advanced maternal age, fibroids, hypertensive disorders, obesity, chorioamnionitis, placenta previa, prolonged or augmented labour, and macrosomia, ${ }^{3-14}$ many cases of postpartum haemorrhage occur in the absence of recognised risk factors, and the pathophysiology that underlies this obstetric complication remains largely unknown.

A genetic predisposition to many important obstetric complications has been established, including pre-eclampsia, ${ }^{15} 16$ preterm birth, ${ }^{17}$ post-term birth, ${ }^{18}$ small for gestational age,${ }^{19}$ and certain congenital malformations. ${ }^{20}$ The possibility of an inherited liability for postpartum haemorrhage has not, to our knowledge, previously been investigated. Understanding the 
extent to which postpartum haemorrhage clusters within families, and how maternal/fetal genetic effects and shared environment contribute to such clustering, could provide new insight into the basis for postpartum haemorrhage.

Making use of nationwide registries of health, we explored familial clustering of postpartum haemorrhage in the Swedish population. We assessed whether clustering could be explained by familial sharing of known risk factors and used the observed cluster correlations to quantify the relative contributions of shared genetic and environmental effects to the liability for postpartum haemorrhage.

\section{Methods}

The unique personal identity number assigned to all Swedish residents allows for linkage among national registers. We linked the medical birth register to the multi-generation register to identify offspring-parent relations. The multi-generation register is constructed around index persons with parental information, through which family structures can be traced. All individuals born since 1932 and registered as residents in Sweden from 1961 onwards are included as index persons in the register. ${ }^{21}$ The medical birth register contains information on $96-99 \%$ of all live births in Sweden since 1973, as well as stillbirths after 28 weeks' gestation. ${ }^{22}$ Other than newborn characteristics (anthropometric measures, APGAR score, etc) the register includes baseline characteristics of the mother such as height, weight before pregnancy, previous illnesses, and smoking. Information on highest attained level of education of the mother was retrieved from the education register at Statistics Sweden. Type of delivery is routinely classified by the midwives as spontaneous, induced vaginal, or caesarean. At the time of discharge, the obstetrician records complications potentially related to pregnancy or delivery using diagnosis codes.

After the implementation of ICD-10 (international classification of diseases, 10th revision) in Sweden in 1997, the threshold for bleeding in the clinical definition of postpartum haemorrhage changed from $>600 \mathrm{~mL}$ to $>1000 \mathrm{~mL}$. Hence, for consistency, we evaluated births after 1997. Further, as family linkage requires the offspring to be included in the multi-generation register we evaluated the outcome only in pregnancies ending with live birth. Postpartum haemorrhage was identified by ICD-10 code O72, which allows specification of timing before $(\mathrm{O} 72.0)$ (such as retained placenta) or after $(\mathrm{O} 72.1)$ the delivery of the placenta. Swedish practice of further classifying the latter according to mechanism (uterine atony (A), lacerations (B), or unknown $(\mathrm{X})$ ) enabled characterisation of aetiological subtypes. During caesarean delivery, haemorrhage is generally coded as a diagnosis of excessive $(>1000 \mathrm{~mL})$ perioperative bleeding (O67.2) without further information on aetiology (atony, retained placenta, etc). As a consequence, we included these as cases in an overall evaluation but focused the analyses only on vaginal deliveries for a more precise classification of postpartum haemorrhage and its subtypes.

\section{Study population}

To boost the information from familial clustering we considered six types of intragenerational relationships - each with a unique expected degree of familial (genetic and environmental) sharing (table $1 \Downarrow$ ). The clustering of births within a person could occur to either intact couples or partner changers (mother/father with different partners) and within siblings to sisters, brothers, or mixed sibling pairs. As the within person clusters imply (at least) two births to each individual, this was made a general inclusion criterion to avoid any systematic differences among clusters.

We first identified all individuals who had at least two children born in Sweden from 1 January 1997 to 31 December 2009 and selected the first two births with the same partner (intact couple) and/or the first two births with separate partners (partner changers). In the latter, we ensured that each individual contributed only once to each cluster. We identified 53292 births to the same mother but separate fathers and 47054 births to the same father but separate mothers. Among all intact couples, we further identified all full siblings, then randomly selected one sibling pair per family. This ensured an individual would contribute only (two births) once to the final sample of 366350 births and the clusters it represented (intact couples and siblings). The pedigree in the figure $\Downarrow$ illustrates one example of the types of clusters a family could contribute, whereby a pair of siblings (here mixed) contributes their first two births to the same partner (two intact couples), and in this example one of the partners also contributes two births as a partner changer (here a mother with separate partners).

From the complete sample of 466686 births, 307756 belonged to clusters in which all deliveries were vaginal. The data were complete for most covariates of interest, with notable exceptions being $9 \%$ missing for maternal height, $12 \%$ for body mass index (BMI) before pregnancy, and 6\% for smoking. For education, country of origin, gestational age, and birth weight each had less than $1 \%$ missing information.

\section{Statistical analyses}

We used three analytical steps to study familial clustering. Firstly, we estimated cluster specific tetrachoric correlations to quantify clustering. We then explored whether clustering could be explained by familial sharing of known risk factors by estimating the cluster specific correlations as pairwise odds ratios in alternating logistic regression models, with and without the inclusion of covariates. Finally we used clustering to quantify the relative contributions (that is, proportion of variation explained) of genetic and environmental effects to the variation in postpartum haemorrhage and modelled these as random effects in a generalised linear mixed model, specifying the expected cluster correlations for each effect.

\section{Familial clustering: tetrachoric correlation}

Postpartum haemorrhage is a multifactorial event that could be assumed to result from an underlying continuous liability to bleed, where only those who reach a certain threshold will experience the event (and be diagnosed). Whenever binary variables can be considered as dichotomisations of a continuous latent trait (whose distribution could be transformed to a standard normal), the correlation between them can be quantified by a tetrachoric correlation coefficient (interpreted like a Pearson correlation coefficient for the latent continuous variable). ${ }^{23}$ Within individuals, clustering was defined by the correlation between the first and second birth. Within siblings, both the siblings' first and second births contributed to clustering.

\section{Influence of risk factors: alternating logistic regression}

To explore the role of known risk factors, we modelled the familial clustering of postpartum haemorrhage using alternating logistic regression. In this procedure, a population mean (where independent variables predict a binary dependent variable) and correlations between observations (clustering of the dependent variable) are estimated simultaneously. Essentially an extension 
of generalised estimating equations, alternating logistic regression produces pairwise odds ratios to describe the correlation within a cluster while at the same time estimating the effect of covariates on the outcome. The pairwise odds ratios express the odds of outcome in relatives (defined by the cluster type) of individuals who experienced the outcome compared with the odds in relatives of individuals without the outcome (reference). Information from the four possible cluster pairings in sibling pairs (odds of first or second birth outcome given sibling's first or second birth outcome) was combined into a joint estimate, as was the information from the two possible intact couple pairings per family (one for each sibling). Clustering was obtained for each unique familial relationship separately, with the ability to simultaneously adjust for covariates that might explain some of the familial clustering. Known potential confounders considered were birth year, maternal age, parity, interval between pregnancies, height, BMI before pregnancy, education, smoking, chronic conditions (diabetes, hypertension, uterine fibroids, coagulopathy), complications of pregnancy (pre-eclampsia and chorioamnionitis), characteristics and complications of delivery (induction of labour, prolonged labour, instrumentally assisted delivery, multiple birth, post-term birth (gestational age $\geq 42$ weeks), and high birth weight ( $\geq 4000 \mathrm{~g}$ ). Each covariate's influence on familial clustering was first explored by comparing the cluster specific pairwise odds ratios from crude and univariable models (complete case); and covariates that influenced the estimate of at least one cluster by more than $1 \%$ were selected to a multivariable adjusted model.

\section{Variance decomposition-generalised linear mixed models}

Familial clustering of postpartum haemorrhage could be a result of shared genetic factors, shared environment, or (most likely) both. By specifying the expected cluster correlations of these components, their contributions to the total variation in postpartum haemorrhage can be assessed from the observed familial clustering by variance decomposition by using a generalised linear mixed model. Table 1 shows the components and their expected correlations specified in the model $\downarrow$. The genetic influence was separated into maternal genetic effects (additive genetic correlation 1 in mother's successive pregnancies and 0.5 in sisters) and/or fetal genetic effects (to which both parents contribute, with additive genetic correlation expected to be 0.5 in intact couples, 0.25 in partner changers, and 0.125 in siblings; table $1 \Downarrow$ ).

Generalised linear mixed models allowed for the total variance in postpartum haemorrhage to be separated into the components specified in table 1, by modelling these as random effects in a probit model. ${ }^{14}{ }^{24}$ The distribution of each effect was assumed normal with mean zero and variances Vmg (maternal genetic), Vf (fetal genetic), Vme (maternal environment), Vc (couple effect), and $\mathrm{Ve}$ (residual variance). This corresponds to a liability-threshold model for postpartum haemorrhage, in which the variance in the underlying liability is assumed possible to transform to a standard normal distribution and partition in different components. By setting Ve equal to

$1-\mathrm{Vmg}-\mathrm{Vf}-\mathrm{Vme}-\mathrm{Vc}$, we modelled the liability as a standard normal distribution, with the variance estimates directly corresponding to variance proportions. Following common quantitative genetic/variance decomposition practice, the model assumes that the genetic and environmental influences on phenotypic variation are additive on the liability scale.

Tetrachoric correlations and alternating logistic regression were performed in SAS software version 9.2 (SAS Institute, Cary,
$\mathrm{NC})$, and generalised linear mixed models were run in R ( $\mathrm{R}$ project for statistical computing, www.r-project.org).

\section{Results}

Among vaginal deliveries, the overall prevalence of postpartum haemorrhage was $4.6 \%$, which was similar to the overall prevalence in the general population during the study period. ${ }^{14}$ Tables 2, 3, and 4 show the distributions of maternal, pregnancy, and delivery characteristics and their relation to postpartum haemorrhage after vaginal delivery (prevalence and crude odds ratios) $\downarrow \Downarrow \Downarrow$, which were similar overall to the full sample that included both vaginal and caesarean deliveries (table A in appendix). Unadjusted odds of postpartum haemorrhage were higher with increasing maternal age, education level, height, and BMI before pregnancy and lower in multiparous mothers and those who smoked (table 2) $\downarrow$. Chronic conditions such as hypertension, coagulopathy (which antedates the delivery), and fibroids were associated with a higher risk of postpartum haemorrhage, as were complications of pregnancy such as gestational diabetes, pre-eclampsia and placenta previa/accreta. Induction and weak/prolonged labour were also clear predictors of postpartum haemorrhage as was multiple birth, post-term birth, and high birth weight (table $4 \Downarrow$ ).

Tetrachoric correlations showed that the strongest clustering occurred in women with same partner (TCC 0.36, 95\% confidence interval 0.34 to 0.38$)$ and new partner $(0.29,0.24$ to 0.35 ; table $5 \Downarrow$ ). In contrast, there was no correlation in postpartum haemorrhage in fathers with separate partners. Weaker correlations were also seen in sisters $(0.10,0.06$ to 0.13$)$ and brothers $(0.05,0.02$ to 0.09$)$ but not in mixed sibling pairs. Inclusion of caesarean deliveries showed a similar pattern, albeit with slightly weaker correlations within individuals (table B in appendix). Clustering in couples can be caused by fetal, maternal genetic, and/or shared "couple" environment, while clustering after a change of partner rather reflects unique maternal and paternal influences (through genes or unique environment). Restriction of outcomes to consider the most common subtypes of postpartum haemorrhage indicated that clustering in women and sisters occurs because of both uterine atony and retained placenta, with the strongest correlations seen for the latter (table $5 \Downarrow)$.

Table $6 \Downarrow$ shows the corresponding cluster correlations expressed as pairwise odds ratios from alternating logistic regression (crude and adjusted). Univariable analysis (table $\mathrm{C}$ in appendix) showed that most covariates had little influence on the estimates of familial clustering: small changes were predominantly seen for the clusters joined by mothers (with the same or new partner, or sister pairs), with high birth weight being the only covariate to reduce either of these pairwise odds ratios by more than $5 \%$ (but still less than 10\%). Simultaneous adjustment for birth cohort, parity, maternal age, height, BMI, induction of labour, complications of labour, post-term birth, and high birth weight resulted in a maximum $10 \%$ drop in the pairwise odds ratios for clusters joined by mothers. Again, results were similar in analyses including all deliveries (see table D in appendix).

Variance decomposition with generalised linear mixed models showed that shared maternal factors explained most of the familial clustering of postpartum haemorrhage after vaginal delivery, with $18 \%$ (95\% confidence interval $9 \%$ to $26 \%$ ) of the total variation attributed to maternal genetic factors and $10 \%$ (1\% to $19 \%$ ) to unique maternal environment (table $7 \Downarrow$ ). Fetal genetic effects were further estimated to explain $11 \%(0 \%$ to $26 \%$ ). With nearly no contribution from couple environment ( $2 \%, 0 \%$ to $9 \%)$, nearly three fifths of the variation in 
postpartum haemorrhage (59\%) could not be explained by factors shared in familial clusters. Inclusion of caesarean deliveries did not materially change these estimates.

\section{Discussion \\ Principal findings}

In this population based study of live births in Sweden, we found that $41 \%$ of the variation in postpartum haemorrhage liability after vaginal delivery was attributable to factors shared in families. The largest fraction of this was attributable to maternal genetic factors (18\% of total variation) and maternal environment present across pregnancies (10\% of total variation). The remaining explained variance was largely attributed to fetal genetic factors (11\% of total variation), the estimate for which, however, did not reach significance. Familial clustering was present for both of the main subtypes of postpartum haemorrhage examined and was strongest for retained placenta. To our knowledge, this is the first report to show a genetic predisposition to postpartum haemorrhage.

Adjustment for known risks factors (obesity, hypertension, diabetes, coagulopathy, etc) only mildly attenuated the estimates of observed familial clustering, suggesting that the observed shared genetic and environmental effects are largely operating through pathways independent of known risk factors. For haemostasis to be achieved after vaginal delivery the placenta must separate from the uterus, the uterus must effectively contract to mechanically constrict the vessels that supplied the placental bed, and coagulation pathways must be activated to form clot at the site of placental attachment. Effective uterine contraction is in turn mediated by the release and effective signalling of oxytocin at the myometrium ${ }^{25}$ It is possible that the genetic variation that confers liability for postpartum haemorrhage acts through one or more of these biological processes. It is also possible that the genetic effects are mediated through pathways that have not been previously implicated in the aetiology of postpartum haemorrhage. Our result should encourage the conduct of studies to identify pathways affected by genetic variation that are associated with postpartum haemorrhage, which could result in new insights into the pathogenesis of this complication.

Together, fetal and maternal genetic effects accounted for $29 \%$ of the variance in liability for postpartum haemorrhage. This estimate of genetic influence is in line with previous findings for preterm birth $(30 \%)^{17}$ and somewhat lower than what has been estimated for post-term birth (47\%), ${ }^{18}$ small for gestational age $(46 \%),{ }^{19}$ and pre-eclampsia $(55 \%) .{ }^{16}$ With nearly a third of the total variation in postpartum haemorrhage liability attributable to genetic effects, and another $10 \%$ explained by the unique maternal environment (shared between pregnancies) and $2 \%$ by couple environment, most of the variation could not be explained by factors shared within families or women across pregnancies. Future research aimed at understanding the aetiology of postpartum haemorrhage, in addition to examining genetic pathways, will need to examine how variations in environment, obstetric management, and other factors contribute to risk.

Given that postpartum haemorrhage is a maternal condition that occurs after the baby has been delivered, it is interesting that our variance decomposition analysis points to a substantial fetal contribution to the variation in liability (albeit with $95 \%$ confidence interval that includes the null). Fetal cells are also involved in placentation, and, as the depth of trophoblast invasion has been suggested as a risk factor for postpartum haemorrhage, ${ }^{25}$ this is a potential mechanism behind the observed fetal genetic effect. Fetal genes, along with maternal genetic factors, are important in many obstetric conditions. ${ }^{16-19}$ Our findings now also suggest that there could be a joint contribution of maternal and fetal genes to postpartum haemorrhage. Our observation of a fetal genetic effect, however, must be tempered by the fact that we did not observe a correlation in men changing partners, and the suggestion of a fetal genetic contribution to postpartum haemorrhage thus derives from the observed small correlation in brother pairs and the lower correlation in women who change partner compared with those who do not. It follows that while our variance decomposition methods point to some fetal genetic influence, this requires cautious interpretation and further study.

In our primary analysis, we focused on vaginal deliveries because postpartum haemorrhage after vaginal delivery is the main source of maternal mortality worldwide; haemorrhage associated with caesarean delivery has a different distribution of aetiologies than in vaginal deliveries and is more often mechanical (such as extension of the hysterotomy or vessel laceration); and the coding of bleeding in connection with caesarean delivery in the medical birth register does not, unlike after vaginal delivery, adequately distinguish the proximate cause (atony, retained placenta, etc). We did, however, also perform all our analyses including caesarean deliveries together with vaginal deliveries and found the same patterns of familial clustering as those observed for vaginal deliveries only, though with somewhat weaker correlations.

Clinically, the familial clustering of postpartum haemorrhage points to the value of assessment of family history in the estimation of risk. Our findings suggest that women with a sister with postpartum haemorrhage had $70 \%$ increased odds of being affected. Assessment of family history in other clinical contexts has proved useful in risk stratification (for example, in cardiac disease and cancer). ${ }^{26}{ }^{27}$ Future studies will need to assess whether this applies to postpartum haemorrhage.

Postpartum haemorrhage is an obstetric emergency that without treatment can lead to maternal death. It might therefore seem surprising that this condition, which affects women of reproductive age and would be expected to decrease these women's reproductive output, could be genetically influenced. Still, in most cases, an event of postpartum haemorrhage implies the secure transmission of maternal genes in at least one surviving offspring. Genes that confer maternal risk of postpartum haemorrhage could also be involved in enhancing fertility by increasing the odds of offspring survival at any point from conception to postnatal health (through the maternal genetic contribution to the offspring genome, and where-at the population level - the death of an individual woman would not be expected to confer decreased survival for her offspring). That fetal genes might influence mother's risk of postpartum haemorrhage could on the other hand be an expression of a "genetic conflict." Applying Trivers' parent-offspring conflict theory ${ }^{28}$ to the setting of pregnancy, Haig stipulated that fetal genes might be selected to draw more resources from the mother than what is optimal to give from the mother's perspective. ${ }^{29}$ Indeed, some have argued that human vulnerability to postpartum haemorrhage is caused by the highly invasive nature of human placentation, which is optimised to secure nourishment to the developing fetus. ${ }^{25}$ Investigation of factors that could improve the fitness of the offspring but increase the tendency of the mother to bleed after delivery might advance the understanding of the biological basis of postpartum haemorrhage. 


\section{Strengths and limitations of the study}

Our study must be interpreted in light of the limitations inherent in its design. While a great strength of the study is that the source population for the cohort included all live births in hospital in Sweden, the extent to which these findings generalise to other populations will need to be established. In particular, the contributions of shared genetic and environmental to liability for postpartum haemorrhage might be different in settings where birth is not medicalised and women are not exposed to drugs like oxytocin or other obstetric interventions. While prior validation work in the Swedish register suggests that the diagnosis is likely to be coded with both sensitivity and specificity, ${ }^{30}$ the outcome assessed reflects physicians' estimates of $>1000 \mathrm{~mL}$ blood loss at the time of delivery, and studies suggest that blood loss is routinely underestimated in the delivery setting. ${ }^{31}{ }^{32}$ Our expectation is that misclassification of estimated blood loss would be non-differential across family members and in women across pregnancies. Assuming that the diagnosis of postpartum haemorrhage is applied with high specificity (which is expected as underestimation of blood loss is far more likely than overestimation), non-differential misclassification of the outcome would, if anything, bias our estimates of familial clustering to the null. An additional potential limitation is that we confined the analysis to a subset of the population (first two births among those with at least two births). The overall prevalence of postpartum haemorrhage in the sample of births included in our analysis, however, is similar to the overall prevalence in the general population during the study period. ${ }^{14}$ The similar prevalences suggest that the subset of the population selected for our analyses were representative of the general population. Furthermore, it would not be possible to estimate the present components of environmental and genetic influence on postpartum haemorrhage without the inclusion of clusters within individuals (producing the requirement of at least two births).

\section{Conclusions and implications}

In conclusion, about a third of the variance in liability for postpartum haemorrhage is attributable to fixed maternal factors. Future research is required to elucidate the pathways involved in mediating the maternal genetic and environmental contributions to risk and to assess the predictive value of considering family history of postpartum haemorrhage when stratifying risk in patients.

Contributors: ASO, BTB, and SH-D were responsible for the original idea, and TF contributed to the design and analyses, which were performed by ASO. All authors contributed to the interpretation of data. ASO and BTB drafted the article. All authors critically revised the article for important intellectual content and approved the final version to be published. ASO is guarantor.

Funding: This study was funded by the Swedish Research Council's international postdoc grant 2012-340 (ASO), research grant 2011-3060 (CA), and through the Swedish Initiative for Research on microdata in the Social and Medical Science (SIMSAM) framework grant 340-2013-5867 (ASO, CA), the strategic research program in epidemiology at Karolinska Institutet (CA), as well as by the Eunice Kennedy Shriver National Institute of Child Health and Human Development of the National Institutes of Health under award No K08HD075831 (BTB). The content is solely the responsibility of the authors and does not necessarily represent the official views of the National Institutes of Health.

Competing interests: All authors have completed the ICMJE uniform disclosure form at www.icmje.org/coi_disclosure.pdf (available on request from the corresponding author) and declare that SDH has received fees from consulting from Novartis and GSK.

Ethical approval: This study was approved by the regional ethical review board in Stockholm, Sweden (DNR 2010-1258-32/5 and 2013/862-31/5).

Transparency statement: ASO affirms that the manuscript is an honest, accurate, and transparent account of the study being reported; that no important aspects of the study have been omitted; and that any discrepancies from the study as planned (and, if relevant, registered) have been explained.

Data sharing: No additional data will be made available.

1 Khan KS, Wojdyla D, Say L, Gulmezoglu AM, Van Look PF. WHO analysis of causes of maternal death: a systematic review. Lancet 2006;367:1066-74.

2 Berg CJ, Callaghan WM, Syverson C, Henderson Z. Pregnancy-related mortality in the United States, 1998 to 2005. Obstet Gynecol 2010;116:1302-9.

3 Oyelese $\mathrm{Y}$, Ananth CV. Postpartum hemorrhage: epidemiology, risk factors, and causes. Clin Obstet Gynecol 2010;53:147-56.

4 Combs CA, Murphy EL, Laros RK Jr. Factors associated with postpartum hemorrhage with vaginal birth. Obstet Gynecol 1991;77:69-76.

5 Sheiner E, Sarid L, Levy A, Seidman DS, Hallak M. Obstetric risk factors and outcome of pregnancies complicated with early postpartum hemorrhage: a population-based study. $J$ Matern Fetal Neonatal Med 2005;18:149-54.

6 Magann EF, Evans S, Hutchinson M, Collins R, Howard BC, Morrison JC. Postpartum hemorrhage after vaginal birth: an analysis of risk factors. South Med J 2005;98:419-22.

7 Kominiarek MA, Kilpatrick SJ. Postpartum hemorrhage: a recurring pregnancy complication. Semin Perinatol 2007;31:159-66.

8 Ford JB, Roberts CL, Bell JC, Algert CS, Morris JM. Postpartum haemorrhage occurrence and recurrence: a population-based study. Med J Aust 2007;187:391-3.

9 Walfish M, Neuman A, Wlody D. Maternal haemorrhage. Br J Anaesth 2009;103(suppl 1):i47-56.

10 Bateman BT, Berman MF, Riley LE, Leffert LR. The epidemiology of postpartum hemorrhage in a large, nationwide sample of deliveries. Anesth Analg 2010;110:1368-73. 1 Blomberg M. Maternal obesity and risk of postpartum hemorrhage. Obstet Gynecol 2011;118:561-8.

12 McLintock C, James AH. Obstetric hemorrhage. J Thromb Haemost 2011;9:1441-51.

13 Ford JB, Shand AW, Roberts CL. Characteristics, causes and treatment of postpartum haemorrhage in first and second pregnancies. Austr N Z J Obstet Gynaecol 2013;53:90-3.

14 Oberg AS, Hernandez-Diaz S, Palmsten K, Almqvist C, Bateman BT. Patterns of recurrence of postpartum hemorrhage in a large, population-based cohort. Am J Obstet Gynecol 2013;210:229.e1-8

15 Lie RT, Rasmussen S, Brunborg H, Gjessing HK, Lie-Nielsen E, Irgens LM. Fetal and maternal contributions to risk of pre-eclampsia: population based study. BMJ 1998;316:1343-7.

16 Cnattingius S, Reilly M, Pawitan Y, Lichtenstein P. Maternal and fetal genetic factors account for most of familial aggregation of preeclampsia: a population-based Swedish cohort study. Am J Med Genet A 2004;130A:365-71.

17 Svensson AC, Sandin S, Cnattingius S, Reilly M, Pawitan Y, Hultman CM, et al. Maternal effects for preterm birth: a genetic epidemiologic study of 630,000 families. Am J Epidemiol 2009;170:1365-72.

18 Oberg AS, Frisell T, Svensson AC, Iliadou AN. Maternal and fetal genetic contributions to postterm birth: familial clustering in a population-based sample of 475,429 Swedish births. Am J Epidemiol 2013;177:531-7.

19 Svensson AC, Pawitan Y, Cnattingius S, Reilly M, Lichtenstein P. Familial aggregation of small-for-gestational-age births: the importance of fetal genetic effects. Am J Obstet Gynecol 2006;194:475-9.

20 Sivertsen A, Wilcox AJ, Skjaerven R, Vindenes HA, Abyholm F, Harville E, et al. Familial risk of oral clefts by morphological type and severity: population based cohort study of first degree relatives. BMJ 2008;336:432-4.

21 Multi-generation register 2009. A description of contents and quality. Statistics Sweden, 2010:1-104.

22 National Board of Health and Welfare. Pregnancies, deliveries and newborn infants-the Swedish medical birth register 1973-2009, assisted reproduction, treatment 1991-2008. [In Swedish.] National Board of Health and Welfare, 2011.

23 Todorov AA, Suarez BK. Genetic liability model. Encyclopedia of biostatistics. John Wiley, 2005.

24 Pawitan Y, Reilly M, Nilsson E, Cnattingius S, Lichtenstein P. Estimation of genetic and environmental factors for binary traits using family data. Stat Med 2004;23:449-65.

25 Abrams ET, Rutherford JN. Framing postpartum hemorrhage as a consequence of human placental biology: an evolutionary and comparative perspective. Am Anthropol 2011;113:417-30.

26 Qureshi N, Armstrong S, Dhiman P, Saukko P, Middlemass J, Evans PH, et al. Effect of adding systematic family history enquiry to cardiovascular disease risk assessment in primary care: a matched-pair, cluster randomized trial. Ann Intern Med 2012;156:253-62.

27 Rubinstein WS, Acheson LS, O'Neill SM, Ruffin MT 4th, Wang C, Beaumont JL, et al. Clinical utility of family history for cancer screening and referral in primary care: a report from the Family Healthware Impact Trial. Genet Med 2011;13:956-65.

28 Trivers RL. Parent-offspring conflict. Am Zool 1974;14:249-64.

29 Haig D. Genetic conflicts in human pregnancy. Q Rev Biol 1993;68:495-532.

30 Swedish medical birth register. A summary of content and quality. Centre for Epidemiology, 2003.

31 Al Kadri HM, Al Anazi BK, Tamim HM. Visual estimation versus gravimetric measurement of postpartum blood loss: a prospective cohort study. Arch Gynecol Obstet 2011;283:1207-13

32 Toledo P, McCarthy RJ, Hewlett BJ, Fitzgerald PC, Wong CA. The accuracy of blood loss estimation after simulated vaginal delivery. Anesth Analg 2007;105:1736-40.

Accepted: 23 July 2014 


\section{What is already known on this topic}

Postpartum haemorrhage is a leading cause of maternal morbidity and mortality

A history of postpartum haemorrhage is a recognised risk factor for haemorrhage in subsequent deliveries

\section{What this study adds}

There is a genetic predisposition to postpartum haemorrhage

About two fifths of the variation in liability for postpartum haemorrhage after vaginal delivery is attributable to factors shared in families, and the largest fraction of this was fixed maternal factors (genes and environment explaining $18 \%$ and $10 \%$ of the total variation, respectively)

Familial clustering occurred for both main subtypes of postpartum haemorrhage (atony and retained placenta), though was most pronounced for retained placenta

Familial sharing of known risk factors could not explain the familial clustering of postpartum haemorrhage

\section{Cite this as: BMJ 2014;349:94984}

This is an Open Access article distributed in accordance with the Creative Commons Attribution Non Commercial (CC BY-NC 3.0) license, which permits others to distribute, remix, adapt, build upon this work non-commercially, and license their derivative works on different terms, provided the original work is properly cited and the use is non-commercial. See: http://creativecommons.org/licenses/by-nc/3.0/. 


\section{Tables}

Table 1| Expected correlations for genetic and environmental effects according to family cluster Sibling pairs joined by Successive births joined by Type of effect (component) Sisters Brothers Mixed Couple Mother Father

\begin{tabular}{lcccccc} 
Maternal genetic effects (Mg) & 0.5 & 0 & 0 & 1 & 1 & 0 \\
\hline Fetal genetic effects (F) & 0.125 & 0.125 & 0.125 & 0.5 & 0.25 & 0.25 \\
\hline Maternal environment (Me) & 0 & 0 & 0 & 1 & 1 & 0 \\
\hline Couple environment (C) & 0 & 0 & 0 & 1 & 0 & 0 \\
\hline Non-shared environment (E) & 0 & 0 & 0 & 0 & 0 & 0
\end{tabular}

Non-shared environment $(\mathrm{E})$ 


\begin{tabular}{|c|c|c|c|}
\hline & Total $^{*}$ & No (\%) with haemorrhage & OR $(95 \% \mathrm{Cl})$ \\
\hline \multicolumn{4}{|l|}{ Age (years) at delivery: } \\
\hline$<20$ & 8847 & $300(3.4)$ & 1.00 \\
\hline $20-34$ & 264294 & $12009(4.5)$ & 1.36 (1.21 to 1.52$)$ \\
\hline$\geq 35$ & 34615 & $1812(5.2)$ & $1.57(1.39$ to 1.78$)$ \\
\hline \multicolumn{4}{|l|}{ Parity: } \\
\hline Primiparous & 136442 & $7169(5.3)$ & 1.00 \\
\hline Multiparous & 171314 & $6952(4.1)$ & $0.76(0.74$ to 0.79$)$ \\
\hline \multicolumn{4}{|l|}{ Civil status: } \\
\hline Living with father & 273137 & $12467(4.6)$ & 1.00 \\
\hline Living alone & 7861 & $321(4.1)$ & $0.89(0.79$ to 1.00$)$ \\
\hline Unknown & 9969 & $398(4.0)$ & $0.87(0.78$ to 0.96$)$ \\
\hline \multicolumn{4}{|l|}{ Country of origin: } \\
\hline Scandinavian & 284545 & $13120(4.6)$ & 1.00 \\
\hline Non-Scandinavian & 23118 & $998(4.3)$ & $0.93(0.87$ to 1.00$)$ \\
\hline \multicolumn{4}{|c|}{ Highest education (years): } \\
\hline Compulsory (9) & 29894 & $1158(3.9)$ & 1.00 \\
\hline Upper secondary $(<3)$ & 48702 & $2082(4.3)$ & $1.11(1.03$ to 1.20$)$ \\
\hline Upper secondary (3) & 88744 & $4140(4.7)$ & $1.21(1.13$ to 1.30$)$ \\
\hline University & 139342 & $6701(4.8)$ & $1.25(1.17$ to 1.34$)$ \\
\hline \multicolumn{4}{|l|}{ Smoking in 1st trimester: } \\
\hline Non-smoker & 258791 & $11984(4.6)$ & 1.00 \\
\hline Smoker & 31295 & $1169(3.7)$ & $0.80(0.75$ to 0.85$)$ \\
\hline \multicolumn{4}{|l|}{ Height $(\mathrm{cm})$ : } \\
\hline$<160$ & 27840 & $1038(3.7)$ & 1.00 \\
\hline 160-169 & 157932 & $6784(4.3)$ & $1.16(1.08$ to 1.24$)$ \\
\hline$\geq 170$ & 102167 & $5223(5.1)$ & $1.39(1.30$ to 1.49$)$ \\
\hline \multicolumn{4}{|l|}{ BMI before pregnancy: } \\
\hline$<20$ & 30910 & $1235(4.0)$ & 1.00 \\
\hline 20-24 & 155186 & $6972(4.5)$ & $1.13(1.06$ to 1.20$)$ \\
\hline$\geq 25$ & 84421 & $4029(4.8)$ & $1.20(1.13$ to 1.29$)$ \\
\hline \multicolumn{4}{|l|}{ Diabetes: } \\
\hline No & 306669 & $14065(4.6)$ & 1.00 \\
\hline Yes & 1087 & $56(5.2)$ & $1.13(0.86$ to 1.49$)$ \\
\hline \multicolumn{4}{|l|}{ Chronic hypertension: } \\
\hline No & 306530 & $14028(4.6)$ & 1.00 \\
\hline Yes & 1226 & $93(7.6)$ & 1.71 (1.38 to 2.12$)$ \\
\hline \multicolumn{4}{|l|}{ Coagulopathy: } \\
\hline No & 306713 & $14048(4.6)$ & 1.00 \\
\hline Yes & 1043 & $73(7.0)$ & $1.57(1.22$ to 2.02$)$ \\
\hline \multicolumn{4}{|l|}{ Fibroids: } \\
\hline No & 307663 & $14110(4.6)$ & 1.00 \\
\hline Yes & 93 & $11(11.8)$ & 2.79 (1.46 to 5.33$)$ \\
\hline
\end{tabular}

*All numbers do not add up to total for all covariates because of missing information: BMI 12\%; height 9\%; smoking 6\%; education, country of origin < $<\%$. 
Table 3| Distribution of postpartum haemorrhage after 307756 Swedish vaginal deliveries (1997-2009) according to characteristics of pregnancy

$$
\text { Total No (\%) with haemorrhage OR }(95 \% \mathrm{Cl})
$$

Gestational diabetes:

\begin{tabular}{lcc}
\hline No 306067 & $14015(4.6)$ & 1.00 \\
\hline Yes 1689 & $106(6.3)$ & $1.40(1.14$ to 1.71$)$ \\
\hline Pre-eclampsia: & & \\
\hline No 301352 & $13546(4.5)$ & 1.00 \\
\hline Yes 6404 & $575(8.9)$ & $2.10(1.92$ to 2.29$)$ \\
\hline Chorioamnionitis: & & \\
\hline No 307539 & $14108(4.6)$ & 1.00 \\
\hline Yes 217 & $13(6.0)$ & $1.33(0.76$ to 2.32$)$ \\
\hline
\end{tabular}

Placenta previa/accrete:

\begin{tabular}{cccc} 
No & 307639 & $14095(4.6)$ & 1.00 \\
\hline Yes & 117 & $26(22.2)$ & $5.95(3.85$ to 9.20$)$ \\
\hline
\end{tabular}

Abruptio placenta:

\begin{tabular}{cccc} 
No & 307616 & $14113(4.6)$ & 1.00 \\
\hline Yes & 140 & $8(5.7)$ & $1.26(0.62$ to 2.57$)$ \\
\hline
\end{tabular}




\begin{tabular}{|c|c|c|c|}
\hline & Total & No (\%) with haemorrhage & OR $(95 \% \mathrm{Cl})$ \\
\hline \multicolumn{4}{|l|}{ Birth year: } \\
\hline $1997-2000$ & 85497 & $3624(4.2)$ & 1.00 \\
\hline 2001-04 & 110811 & $5105(4.6)$ & 1.09 (1.04 to 1.14$)$ \\
\hline $2005-09$ & 111448 & $5392(4.8)$ & 1.15 (1.10 to 1.20$)$ \\
\hline \multicolumn{4}{|l|}{ Induction: } \\
\hline No & 274586 & $11896(4.3)$ & 1.00 \\
\hline Yes & 33170 & $2225(6.7)$ & 1.59 (1.51 to 1.66$)$ \\
\hline \multicolumn{4}{|c|}{ Weak/prolonged labour: } \\
\hline No & 264200 & $11190(4.2)$ & 1.00 \\
\hline Yes & 43556 & $2931(6.7)$ & $1.63(1.56$ to 1.70$)$ \\
\hline \multicolumn{4}{|l|}{ Sex of baby: } \\
\hline Male & 157804 & $6996(4.4)$ & 1.00 \\
\hline Female & 149952 & $7125(4.8)$ & $1.08(1.04$ to 1.11$)$ \\
\hline \multicolumn{4}{|l|}{ Multiples: } \\
\hline Single birth & 305778 & $13880(4.5)$ & 1.00 \\
\hline Multiples & 1978 & $241(12.2)$ & 2.92 (2.55 to 3.34$)$ \\
\hline \multicolumn{4}{|l|}{ Post-term: } \\
\hline No & 292334 & $12952(4.4)$ & 1.00 \\
\hline Yes & 15173 & $1158(7.6)$ & $1.78(1.67$ to 1.90$)$ \\
\hline \multicolumn{4}{|c|}{ High birth weight $(\geq 4000 \mathrm{~g})$ : } \\
\hline No & 245155 & $9764(3.9)$ & 1.00 \\
\hline Yes & 61785 & $4336(7.0)$ & 1.82 (1.75 to 1.89$)$ \\
\hline
\end{tabular}

*All numbers do not add up to total for all covariates because of missing information: gestational age and birth weight $<1 \%$ ). 
Table 5 | Clustering of postpartum haemorrhage after vaginal delivery. Figures are tetrachoric correlations (TCC) and $95 \%$ confidence intervals

TCC $(95 \% \mathrm{Cl})$

Cluster level No of pairwise correlations All vaginal deliveries Uterine atony Retained placenta

\begin{tabular}{lcccc} 
Couples & 116552 & $0.36(0.34$ to 0.38$)$ & $0.30(0.26$ to 0.33$)$ & $0.45(0.42$ to 0.48$)$ \\
\hline Mothers, new partner & 21090 & $0.29(0.24$ to 0.35$)$ & $0.22(0.13$ to 0.31$)$ & $0.37(0.28$ to 0.46$)$ \\
\hline Fathers, new partner & 16236 & $0(-0.07$ to 0.07$)$ & $-0.02(-0.16$ to 0.12$)$ & $0.04(-0.09$ to 0.18$)$ \\
\hline Sisters & 63352 & $0.10(0.06$ to 0.13$)$ & $0.05(-0.01$ to 0.11$)$ & $0.09(0.02$ to 0.17$)$ \\
\hline Brothers & 57332 & $0.05(0.02$ to 0.09$)$ & $0(-0.07$ to 0.07$)$ & $0.05(-0.03$ to 0.13$)$ \\
\hline Mixed siblings & 112420 & $0.01(-0.02$ to 0.03$)$ & $-0.03(-0.08$ to 0.02$)$ & $0(-0.06$ to 0.06$)$ \\
\hline
\end{tabular}


Table 6 | Clustering of postpartum haemorrhage after vaginal delivery. Figures are pairwise odds ratios (POR) and $95 \%$ confidence interval

\begin{tabular}{|c|c|c|c|c|c|}
\hline \multirow[b]{2}{*}{ Cluster level } & \multicolumn{2}{|c|}{ All vaginal deliveries } & \multicolumn{3}{|c|}{ Vaginal deliveries-complete case } \\
\hline & $\begin{array}{l}\text { No of pairwise } \\
\text { correlations }\end{array}$ & Crude POR $(95 \% \mathrm{Cl})$ & $\begin{array}{l}\text { No of pairwise } \\
\text { correlations }\end{array}$ & Crude POR $(95 \% \mathrm{Cl})$ & Adjusted* POR (95\% Cl) \\
\hline Couples & 116552 & 4.54 (4.20 to 4.91$)$ & 91462 & 4.75 (4.35 to 5.19$)$ & 4.28 (3.90 to 4.70$)$ \\
\hline Mothers, new partner & 21090 & 3.64 (2.93 to 4.51$)$ & 18335 & 3.35 (2.63 to 4.26$)$ & 3.03 (2.37 to 3.89 ) \\
\hline Fathers, new partner & 16236 & 0.97 (0.69 to 1.37$)$ & 13921 & $1.03(0.71$ to 1.48$)$ & 1.00 (0.69 to 1.45$)$ \\
\hline Sisters & 63352 & $1.58(1.34$ to 1.87$)$ & 49828 & 1.55 (1.27 to 1.88$)$ & $1.46(1.20$ to 1.78$)$ \\
\hline Brothers & 57332 & 1.27 (1.05 to 1.53$)$ & 45072 & $1.21(0.96$ to 1.51$)$ & $1.17(0.93$ to 1.48$)$ \\
\hline Mixed siblings & 112420 & 1.03 (0.89 to 1.19$)$ & 88024 & 1.05 (0.89 to 1.24$)$ & 1.03 (0.87 to 1.22$)$ \\
\hline
\end{tabular}

*Adjusted includes birth year, maternal age, parity, interval between pregnancies, height, BMI, induction, labour, complications, post-term birth, and high birth weight. 
Table $7 \mid$ Genetic and environmental contribution to the variation in postpartum haemorrhage. Figures are percentage of explained variation with $95 \%$ confidence intervals

Effect Vaginal deliveries Vaginal and caesarean deliveries

Maternal genetic effect $\quad 18$ (9 to 26$) \quad 18$ (11 to 22$)$

Fetal genetic effect $\quad 11$ (0 to 26$) \quad 12$ (1 to 23 )

Unique maternal environment $10(1$ to 19$) \quad 6(0$ to 13$)$

$\begin{array}{lll}\text { Couple effect } & 2(0 \text { to } 9) & 1(0 \text { to } 6)\end{array}$ 


\section{Figure}

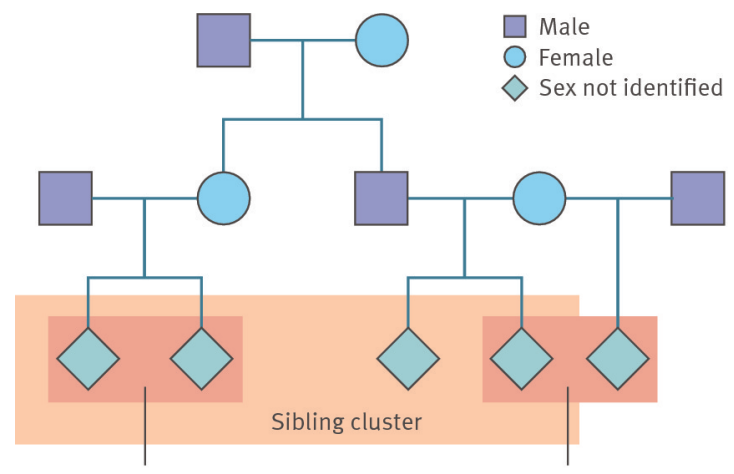

Intact couple cluster

Partner changer cluster

Family pedigree illustrating example of types of clusters (intact couple, partner changer, and sibling) used to assess familial clustering of postpartum haemorrhage 